\title{
ON A REPRESENTATION OF THE AUTOMORPHISM GROUP OF A GRAPH IN A UNIMODULAR GROUP
}

\author{
ISTVÁN ESTÉLYI, JÁN KARABÁŠ, ROMAN NEDELA, AND ALEXANDER MEDNYKH
}

\begin{abstract}
We investigate a representation of the automorphism group of a connected graph $X$ in the group of unimodular matrices $U_{\beta}$ of dimension $\beta$, where $\beta$ is the Betti number of graph $X$. We classify the graphs for which the automorphism group does not embed into $U_{\beta}$. It follows that if $X$ has no pendant vertices and $X$ is not a simple cycle, then the representation is faithful and Aut $X$ acts faithfully on $H_{1}(X, \mathbb{Z})$. The latter statement can be viewed as a discrete analogue of a classical Hurwitz's theorem on Riemann surfaces of genera greater than one.
\end{abstract}

\section{INTRODUCTION}

Given any topological space $X$ and a finite group $G$ of homeomorphisms of $X$, consider the associated action of $G$ on the first homology group $H_{1}(X, \mathbb{Z})$. It is natural to ask when this representation is faithful. Hurwitz's theorem says when $X$ is a Riemann surface of genus greater than one, the answer is yes, see Farkas and Kra [4, Theorem V.3.1, p. 270]. A. M. MacBeath in [6] proved that the action remains faithful on the homology group $H_{1}\left(X, \mathbb{Z}_{p}\right), p>2$, but fails for $H_{1}\left(X, \mathbb{Z}_{2}\right)$, see also [4, p. 276]. In this paper we consider the case where $X$ is a finite graph. A motivation to investigate it comes from the applications of the matrix representation of the automorphism group of a graph in the lifting automorphism problem [7, 8, 9]. Recently we have found an application of the result in investigation of structure of Jacobian of a graph, see [1] for definitions and further details.

Throughout we assume that $X$ is a simple connected graph. By a dart of $X$ we mean an edge endowed with one of the two possible orientations. Thus every edge $u v$ gives rise to two darts $(u, v)$ and $(v, u)$ distinguished just by the orientation. If $x$ is dart, then the oppositely oriented dart underlying the same edge will be denoted by $x^{-1}$. An unoriented simple cycle is a connected subgraph of $X$, where every vertex has degree 2 . Any simple cycle has two orientations $C, C^{-1}$ which we call oriented (simple) cycles.

Let $T$ be a spanning tree of $X$. For every co-tree edge $e_{i}$ choose one of the two underlying darts, and denote it by $x_{i}$. Clearly, each $x_{i}$ determines a unique simple oriented cycle $C_{i}=\left(x_{i}, y_{1}, y_{2}, \ldots, y_{k}\right)$, where $y_{1} y_{2} \ldots y_{k}$ is the unique path in $T$ joining the terminal vertex of $x_{i}$ to the initial vertex of $x_{i}$. The cycles $C_{i}$, $i=1, \ldots, \beta$ will be called fundamental oriented cycles. They form a basis of first homology group $H_{1}(X)$ which is isomorphic to free abelian group of rank $\beta$, where $\beta(X)$ is the Betti number.

2010 Mathematics Subject Classification. 05C25 (Primary), 20F65, 20C10, 20H25 (Secondary).

Key words and phrases. graph, automorphism, unimodular matrix. 
An automorphism of a graph is a permutation of the vertices taking adjacent vertices onto adjacent vertices. In this note we consider as a rule the induced action of graph automorphisms on the set of darts. Hence automorphisms of a graph are considered as permutations of the set of darts. For every automorphism $f \in$ Aut $X$ we can construct a matrix $M=M_{T}(f)$ with entries in $\{1,0,-1\}$ as follows. Fix a linear order of the co-tree edges $e_{1}, e_{2}, \ldots, e_{\beta}$, where $\beta=\beta(X)=e(X)-v(X)+1$. The automorphism $f$ of the graph $X$ induces a linear transformation of $H_{1}(X)$ which we can treat as a vector space of dimension $\beta$ (with integer scalars). Then $M_{T}(f)$ is the usual matrix of a linear transformation with respect to the basis given by the fundamental oriented cycles $C_{i}$ determined by co-tree darts of $T$. In particular, the entry $m_{i, j}$ of $M_{T}(f)$ takes value 1 or -1 if and only if $x_{i}$ or $x_{i}^{-1}$ is a dart traversed by $f\left(C_{j}\right)$, respectively. Finally, choosing a different spanning tree $T^{\prime}$ can be viewed as a change of basis of the vector space $H_{1}(X)$. Hence, $M_{T^{\prime}}(f)$ is a conjugate of $M_{T}(f)$ by a matrix representing the coordinates for the new cycles in terms of the old cycles. In particular, the issue of faithfulness is obviously independent of the choice of spanning tree.

Recall that a square matrix is unimodular if its determinant is \pm 1 . The unimodular matrices of dimension $\beta$ (with standard multiplication) form a group, here denoted $U_{\beta}$. By convention, if $\beta=0$, the group $U_{\beta}$ is trivial. The following result is well-known.

Theorem $1([9])$. The assignment $\Theta_{T}: f \mapsto M_{T}(f)$ defines a homomorphism of Aut $X$ into the group of unimodular matrices.

Observe that if $X$ is a tree with a non-trivial automorphism group, or if $X$ is a simple cycle, then $\Theta=\Theta_{T}$ is not injective for any spanning tree $T$ of $X$. In what follows, we consider the following problem:

Problem. For which connected graphs $X$ does the homomorphism $\Theta_{T}$ determine an embedding of Aut $X$ into the group $U_{\beta}$ ?

\section{Main Result}

We will see that the answer to the posed problem depends on the structure of blocks of $X$. To this end, we recall a few related basic results on the 2-connectivity of graphs, that can be found e.g. in monographs [2, Chapter 5] and [3, Chapter 3]. A graph with at least two vertices is 2-connected, if it cannot be disconnected by the removal of a single vertex, or in other words, if it has no cutvertices. A bridge in a connected graph is an edge whose removal disconnects the graph. A bridgeless connected graph is 2-edge-conected. Clearly, a 2-connected graph is 2edge-connected.

By a block of a graph $X$ we mean a maximal 2-connected induced subgraph of $X$. A block with at least three vertices is called nontrivial. Blocks of an arbitrary simple graph determine a decomposition of the set of edges. More precisely, the following properties hold true:

(P1) any two blocks of $X$ have at most one vertex in common,

(P2) every edge of $X$ belongs to exactly one block of $X$,

(P3) each cycle of $X$ is contained in exactly one block of $X$.

The block tree $\mathcal{B}=\mathcal{B}(X)$ of a connected graph $X$ is a bipartite graph with the vertex set $V(\mathcal{B})$ formed by the blocks of $X$ and by the cutvertices of $X$. A cutvertex $v \in V(\mathcal{B})$ is adjacent to a block $B \in V(\mathcal{B})$ if and only if $v \in B$. It can be easily seen 
that $\mathcal{B}$ is a tree [3, Proposition 3.1.2]. All leafs of $\mathcal{B}$ are blocks. It is well-known [3, Chapter 3] that the centre of $\mathcal{B}$ is a single vertex, that can either be a block or a cutvertex of $X$. In case it is a block, it is called the central block of $X$. Since an automorphism $f \in$ Aut $X$ maps blocks onto blocks and permutes the cutvertices, $f$ induces an automorphism $f^{*}$ of $\mathcal{B}(X)$. Clearly, $f^{*}$ fixes the central vertex of $\mathcal{B}$ and $f^{*}(v)=f(v)$ if $v$ is a cutvertex.

Lemma 2. Let $T$ be an arbitrary spanning tree of $X$, let $C_{i} \neq C_{j}$ be fundamental oriented cycles with respect to $T$ and let $f \in \operatorname{ker} \Theta_{T}$. Then $C_{i}, i=1,2, \ldots, \beta(X)$ are fixed by $f$ setwise. Moreover, if $C_{i} \cap C_{j} \neq \emptyset$, then $C_{i} \cup C_{j}$ is fixed pointwise.

Proof. Since $M_{T}(f)=\mathrm{id}, f\left(C_{i}\right)$ traverses exactly one co-tree dart $x_{i}$ determining $C_{i}$. Thus $f\left(C_{i}\right)=C_{i}$. Assume $C_{i} \cap C_{j}$ is a path $P$, possibly of length zero. By the previous statement, $P$ is fixed pointwise. Clearly, there exist a dart in $C_{i}-P$ incident to an endvertex of $P$. Such a dart is fixed by $f$. An automorphism of a simple cycle $C$ fixing a dart fixes $C$ pointwise.

Corollary 3. If $f \in \operatorname{ker} \Theta_{T}$, then $f(B)=B$ for each nontrivial block $B$ in $X$.

Lemma 4. A maximal 2-edge-connected subgraph of a connected graph $X$ that is not a simple cycle is fixed pointwise by $f \in$ Aut $X$.

Proof. Let $Z$ be a subgraph of $X$ satisfying the assumptions. Let $Y$ be a graph whose vertices are oriented fundamental cycles of $Z$. Two such cycles are adjacent if they have nontrivial intersection. Since $Z$ has no bridges, $Y$ is connected. By the assumptions $Y$ contains more than one vertex. Using induction based on Lemma 2, $f$ is the identity on $Z$.

Proposition 5. Let $X$ be a simple connected graph without vertices of degree one. Then either the homomorphism $\Theta_{T}$ : Aut $X \rightarrow U_{\beta}$ is injective or $X$ is a simple cycle.

Proof. If $X$ is 2-edge-connected, the statement follows from Lemma 4.

Suppose now that $X$ has bridges. Consider the block tree $\mathcal{B}=\mathcal{B}(X)$. By assumptions, $\mathcal{B}(X)$ has at least 3 vertices. Since there are no vertices of degree one, every leaf in $\mathcal{B}$ represents a nontrivial block. By Corollary 3, every leaf of $\mathcal{B}(X)$ is fixed. Since any automorphism of a tree fixing all the leafs is the identity, we have $f^{*}=$ id. Hence, $f$ fixes all bridges of $X$. By Lemma $4, f$ also fixes blocks of $X$ that are not cycles pointwise. If a block $B$ is a cycle and $X$ has at least two blocks, then the unique simple path $P$ in $\mathcal{B}$ from $B$ to any block $B^{\prime}$ is fixed by $f$. By definition of $\mathcal{B}, P$ traverses a cutvertex $u \in B$. Since $f$ fixes $u \in B$, by Lemma $2,\left.f\right|_{B}=$ id. Therefore, $f=$ id on $X$.

Let $w \in V(X)$ be a cutvertex of $X$ such that there exist a bridge $B_{1}$ and a nontrivial block $B_{2} ¥ K_{2}$, both containing $w$. By a pendant tree of $X$ rooted at $w$ we mean a subgraph of $X$ induced by $\{w\} \cup V\left(F_{w}\right)$, where $F_{w}$ is the maximal acyclic subgraph of $X-w$. A pendant tree will be called rigid, if its automorphism group is trivial. A unicyclic graph will be called periodic if it admits a non-trivial automorphism $f$ rotating the unique cycle.

Example 6. All periodic unicyclic graphs can be constructed as follows. Choose two positive integers $n, k$ such that $n>2, k \mid n$, and $k<n$. Further, choose a sequence of rooted trees $S_{0}, S_{1}, \ldots, S_{k-1}$ with the corresponding roots $w_{0}, w_{1}, \ldots, w_{k-1}$. 
Let $\mathbf{C}=\left(v_{0}, v_{1}, \ldots, v_{n-1}\right)$ be a simple cycle with vertices $v_{i}, i \in\{0,1, \ldots, n-1\}$. Form a graph $X$ by taking the cycle $\mathbf{C}$ and attach to every vertex $v_{j}$ of $\mathbf{C}$ a copy of the tree $S_{i}$, where $j \equiv i(\bmod k)$, by identifying $v_{j}$ with the root $w_{i}$ of $S_{i}$, see e.g. Figure 1.

It is obvious that $X$ admits an automorphism $\varrho$ of order $\frac{n}{k}>1$ rotating $\mathbf{C}$ with period $k$. Hence, $\varrho$ is a nontrivial element in the kernel of $\Theta_{T}$, for any spanning tree $T$ of $X$. Therefore, $\Theta_{T}$ is not injective.
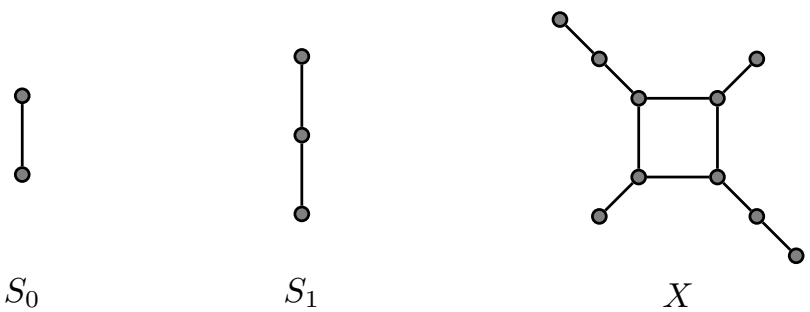

Figure 1. An example of a periodic unicyclic graph $X$ with $n=4$ and $k=2$.

Now we are ready to prove the main result.

Theorem 7. Let $X$ be a connected simple graph and let $T$ be a spanning tree of $X$. Then $\Theta_{T}$ is not injective if and only if at least one of the following statements holds.

(i) $X$ is a tree and Aut $X \neq 1$,

(ii) $X$ contains a pendant tree $S$ such that Aut $S \neq 1$,

(iii) $X$ is periodic unicyclic.

Proof. The direction $(\Leftarrow)$ is straightforward. If $X$ is a tree, then $\beta(X)=0$ and $U_{\beta}$ is trivial, hence $\Theta_{T}$ is not injective whenever Aut $X \neq 1$. If (ii) holds, then there exists an automorphism $\tau \in \operatorname{ker} \Theta_{T}$ fixing the complement of the pendant tree $S$ pointwise and acting nontrivially on $S$. The case (iii) is treated in Example 6 .

$(\Rightarrow)$ In order to derive a contradiction we assume that there exists a nontrivial automorphism $f \in \operatorname{ker} \Theta_{T}$ and none of (i), (ii), or (iii) holds true. In particular, we assume that $X$ is not a tree.

If $X$ contains some pendant trees $S_{0}, S_{1}, \ldots, S_{k-1}$, we reduce $X$ to a graph $X^{\prime}$ by removing the trees $S_{0}, \ldots, S_{k-1}$. Since each of $S_{i}$ is rigid, we have ker $\left.\Theta_{T}\right|_{X^{\prime}} \geq$ ker $\Theta_{T}$. By Proposition 5 ker $\left.\Theta_{T}\right|_{X^{\prime}}$ is trivial.

\section{Concluding Remarks}

The aim of this section is to discuss several problems related to the main result. The first problem reads as follows.

Problem 1. What is the smallest dimension $d$ such that Aut $X$ is faithfully represented by $d \times d$ unimodular matrices?

Theorem 7 characterises graphs $X$ for which $d \leq \beta(X)$. On the other hand, the automorphism group of a complete graph $K_{n}, n>3$, Aut $K_{n} \cong \mathrm{S}_{n}$, is faithfully represented by permutation matrices of type $n \times n$, while $\beta\left(K_{n}\right)=(n-1)(n-2) / 2$. 
The other extreme is a cycle $C_{p}, p$ is prime, which automorphism group Aut $C_{p} \cong$ $\mathrm{D}_{p}$, with faithful representation by $p \times p$ matrices while $\beta\left(C_{p}\right)=1$.

Another motivation comes from representation of groups. Given a group $G$ one can use Frucht's theorem [5] and ask for the graph $X$ such that Aut $X \cong G$. One can ask for 'the smallest unimodular representation' of the group $G$.

Problem 2. Given a finite group $G$ find the smallest graph $X$ such that $G \leq$ Aut $X$ is faithfully represented by unimodular matrices.

One can take $X$ to be a Cayley graph for $G$ and then ask the following.

Problem 3. What is the smallest Betti number $\beta(X)$ through all Cayley graphs $X$ for $G$ such that the representation of Aut $X=G$ is faithful?

The question is related as well to the problem how to find the smallest generating set of $G$, giving the smallest valence of $X$.

A short analysis of our argumentation shows that the proof of faithfulness of the unimodular representation of Aut $X$ does not impose any requirements on the field over which the matrices are considered, except that $1 \neq-1$. It follows that the representation remains faithful if the matrices $M_{T}(f)$ are considered over fields of characteristic $p>2$.

\section{ACKNOWLEDGEMENTS}

The authors express thanks to the anonymous referee for his/her useful comments which helped a lot to improve the presentation.

The first three authors were supported by the grant GACR 20-15576S. The first author acknowledges the financial support of Széchenyi 2020 under the EFOP3.6.1-16-2016-00015 grant. The second and third author were supported by the grant No. APVV-19-0308 of Slovak Research and Development Agency. The fourth author was supported by Mathematical Center in Akademgorodok under agreement No. 075-15-2019-1613 with the Ministry of Science and Higher Education of the Russian Federation.

\section{REFERENCES}

[1] M. Baker and S. Norine. Riemann-Roch and Abel-Jacobi theory on a finite graph. Advances in Mathematics, 215(2):766-788, 2007.

[2] A. Bondy and U. Murty. Graph Theory, volume 244 of Graduate Texts in Mathematics. Springer Verlag, London, 2011.

[3] R. Diestel. Graph Theory, volume 101 of Graduate Texts in Mathematics. Springer Verlag, New York, 2005.

[4] H. Farkas and I. Kra. Riemann Surfaces, volume 71 of Graduate Texts in Mathematics. Springer Verlag, New York, 2nd edition, 1992.

[5] R. Frucht. Herstellung von graphen mit vorgegebener abstrakter gruppe. Compositio Mathematica, 6:239-250, 1939.

[6] A. M. MacBeath. Action of Automorphisms of a Compact Riemann Surface on the First Homology Group. Bulletin of the London Mathematical Society, 5(1):103-108, 1973.

[7] A. Malnič, D. Marušič, and P. Potočnik. Elementary abelian covers of graphs. Journal of Algebraic Combinatorics, 20(1):71-97, 2004.

[8] A. Malnič, R. Nedela, and M. Škoviera. Lifting graph automorphisms by voltage assignments. European Journal of Combinatorics, 21(7):927-947, 2000.

[9] J. Širán̆. Coverings of graphs and maps, orthogonality, and eigenvectors. Journal of Algebraic Combinatorics: An International Journal, 14(1):57-72, 2001. 
(I. Estélyi) NTIS, University of West Bohemia, Technická 8, 30100 Plzeñ 3, Czech REPUBLIC

(I. Estélyi) Faculty of Information Technology, University of Pannonia, Egyetem u. 10., 8200 Veszprém, Hungary

Email address, I. Estélyi: estelyi.istvan@mik.uni-pannon.hu

(J. Karabáš) Faculty of Natural Sciences, Matej Bel University, Tajovského 40, 97401 BAnská Bystrica, Slovakia

(J. Karabáš) NTIS, University of West Bohemia, Technická 8, 30100 Plzeñ 3, Czech REPUBLIC

Email address, J. Karabáš: karabas@savbb.sk

(R. Nedela) Faculty of Applied Sciences, University of West Bohemia, Technická 8, 30100 PlzeŇ 3, CZeCh Republic

(R. Nedela) Mathematical Institute, Slovak Academy of Sciences, Ďumbierska 1, 97411 Banská Bystrica, SlOVAKIA

Email address, R. Nedela: nedela@ntis.zcu.cz

(A. Mednykh) Sobolev Institute of Mathematics, Pr. Koptyuga 4, Novosibirsk, 630090, RUSSIA

(A. Mednykh) Novosibirsk State University Pirogova 2, Novosibirsk, 630090, Russia

Email address, A. Mednykh: smedn@mail.ru 